\title{
FUNCTIONS WITH DIFFERENT STRONG AND WEAK Ф-VARIATIONS
}

\author{
LANE YODER
}

\begin{abstract}
This paper shows by example how different the strong $\Phi$ variation can be from the weak $\Phi$-variation. Let $\Phi$ be a convex function on $[0, \infty)$ with $\Phi(0)=0$. A continuous function $f$ on $[a, b]$ is of bounded strong $\Phi$-variation if sup $\sum \Phi\left(\left|f\left(x_{i}\right)-f\left(x_{i-1}\right)\right|\right)<\infty$, for the partitions of $[a, b]$. Since inf $\sum \Phi\left(\left|f\left(x_{i}\right)-f\left(x_{i-1}\right)\right|\right)=0$ if $\lim _{x \rightarrow 0} x^{-1} \Phi(x)=0$, the weak $\Phi$ variation is defined as inf $\sum \Phi\left(\omega\left(f ; x_{i-1}, x_{i}\right)\right)$, where $\omega(f ; c, d)$ is the oscillation of $f$ on $[c, d]$. Of special interest is the case $\Phi(x)=x^{p}, p \geqslant 1$, in terms of which strong and weak variation dimensions are defined. They are denoted by $\operatorname{dim}_{\mathrm{s}}(f)$ and $\operatorname{dim}_{\mathrm{w}}(f)$, respectively. By a lemma of Goffman and Loughlin, the Hausdorff dimension $d$ of the graph of $f$ provides a lower bound for $\operatorname{dim}_{w}(f): 1 /(2-d) \leqslant \operatorname{dim}_{w}(f)$. A Lipschitz condition of order $\alpha$ provides an upper bound for $\operatorname{dim}_{s}(f): \operatorname{dim}_{s}(f) \leqslant 1 / \alpha$. Besicovitch and Ursell showed that $1 \leqslant d \leqslant 2-\alpha$ and gave examples to show that $d$ can take on any value in this interval. It turns out that these examples provide the extreme cases for variation dimensions; i.e., $\operatorname{dim}_{w}(f)=1 /(2-d)$ and $\operatorname{dim}_{\mathbf{s}}(f)=1 / \alpha$.
\end{abstract}

1. Introduction. The purpose of this paper is to show by example how different the strong variation of a function can be from the weak variation. In previous work concerning the variation of Brownian motion, the strong and weak variation dimensions were shown to be equal.

Strong and weak $\Phi$-variations were defined by Goffman and Loughlin [2]. If $\Phi$ is a nonnegative convex function on $[0, \infty)$ with $\Phi(0)=0$, and $f$ is a real function on $[a, b]$, the strong $\Phi$-variation of $f$ is

$$
V_{\Phi}^{\mathrm{s}}(f)=\sup _{\pi} \sum_{i=1}^{n} \Phi\left(\left|f\left(x_{i}\right)-f\left(x_{i-1}\right)\right|\right),
$$

where $\pi$ is any partition $a=x_{0}<\cdots<x_{n}=b$ of $[a, b]$. As Goffman and Loughlin pointed out, if $\lim _{x \rightarrow 0} x^{-1} \Phi(x)=0$, then, for every continuous $f$,

$$
\inf _{\pi} \sum_{i=1}^{n} \Phi\left(\left|f\left(x_{i}\right)-f\left(x_{i-1}\right)\right|\right)=0 .
$$

This fact motivates the definition of weak $\Phi$-variation,

$$
V_{\Phi}^{\mathrm{w}}(f)=\inf _{\pi} \sum_{i=1}^{n} \Phi\left(\omega\left(f ; x_{i-1}, x_{i}\right)\right),
$$

where

Presented to the Society, January 20, 1975; received by the editors March 11, 1975. AMS (MOS) subject classifications (1970). Primary 26A45. 


$$
\omega(f ; c, d)=\sup _{c \leqslant x \leqslant y \leqslant d}|f(x)-f(y)| .
$$

Using $\Phi(x)=x^{p}, p \geqslant 1$, the $\Phi$-variations of $f$ will be called the strong $p$ variation, $V_{p}^{\mathrm{s}}(f)$, and the weak $p$-variation, $V_{p}^{\mathrm{w}}(f)$. Then either $V_{p}^{\mathrm{s}}(f)=\infty$ for all $p \geqslant 1, V_{p}^{s}(f)<\infty$ for all $p>1$, or there is a unique $p>1$ such that $V_{q}^{\mathrm{s}}(f)=\infty$ for all $q<p$ and $V_{q}^{\mathrm{s}}(f)<\infty$ for all $q>p$. This yields the strong variation dimension of $f, \operatorname{dim}_{\mathrm{s}}(f)$, which is $\infty, 1$, and $p$, respectively, in the three cases. Similarly, the weak variation dimension of $f, \operatorname{dim}_{\mathrm{w}}(f)$ is defined according to $V_{p}^{\mathrm{w}}(f)>0$ or $V_{p}^{\mathrm{w}}(f)=0$.

Note. Goffman and Loughlin defined the weak $\Phi$-variation as lim inf $\sum \Phi\left(\omega\left(f ; x_{i-1}, x_{i}\right)\right)$ as the norms of the partitions converge to zero. The weak $\Phi$-variation given here leaves $\operatorname{dim}_{\mathrm{w}}(f)$ unchanged and was so defined simply because it more closely parallels the definition of strong variation.

Goffman and Loughlin showed that both the strong and weak variation dimensions of Brownian motion are two with probability one. At the suggestion of Professor Goffman I extended the concepts of strong and weak variation to higher dimensions and showed that with appropriate definitions, $N$-parameter Brownian motion in $d$-space has strong and weak variation dimensions $2 N$ with probability one [4], [5]. Taylor [3] found precise functions $\Phi$ for measuring the strong and weak $\Phi$-variations of Brownian motion.

The Lipschitz condition of a function gives an upper bound for the strong variation dimension, and the Hausdorff dimension of the graph gives a lower bound for the weak variation dimension. The examples given here, taken from Besicovitch and Ursell [1], are the extreme cases; that is, they have the largest possible strong variation dimension and the smallest possible weak variation dimension.

2. Preliminary lemmas. It is clear from the definitions that for every function $f, V_{p}^{\mathrm{w}}(f) \leqslant V_{p}^{\mathrm{s}}(f)$, and therefore

$$
\operatorname{dim}_{w}(f) \leqslant \operatorname{dim}_{\mathbf{s}}(f) .
$$

A function $f$ on $[0,1]$ satisfies a Lipschitz condition of order $\delta(f \in \operatorname{Lip} \delta)$ if

$$
M_{f}=\sup _{x \neq y}|f(x)-f(y)||x-y|^{-\delta}<\infty .
$$

The following lemma shows that if $f \in \operatorname{Lip} \delta$, then

$$
\operatorname{dim}_{s}(f) \leqslant 1 / \delta .
$$

Lemma 1. If $f \in \operatorname{Lip} \delta$, then $V_{1 / \delta}^{\mathrm{s}}(f)<\infty$.

Proof. For any partition $0=x_{0}<x_{1}<\cdots<x_{n}=1$,

$$
\sum_{i=1}^{n}\left|f\left(x_{i}\right)-f\left(x_{i-1}\right)\right|^{1 / \delta} \leqslant \sum_{i=1}^{n}\left[M_{f}\left(x_{i}-x_{i-1}\right)^{\delta}\right]^{1 / \delta}=M_{f}^{1 / \delta} .
$$

The following lemma, due to Goffman and Loughlin [2], shows that if the Hausdorff dimension of the graph of $f$ is $d$, then

$$
1 /(2-d) \leqslant \operatorname{dim}_{\mathrm{w}}(f) .
$$


LEMMa 2. If $V_{p}^{\mathrm{w}}(f)=0$, then $H_{2-1 / p}(\mathrm{gr} f)=0$. (Here $H_{k}$ is the Hausdorff $k$ dimensional measure and $\mathrm{gr} f$ is the graph of $f$.)

We note here that Goffman and Loughlin stated the lemma in a different form, but the different definition of weak $\Phi$-variation does not affect the validity of Lemma 2 . All that is required for the proof of the lemma is a sequence of partitions of $[a, b]$, with norms converging to zero, which gives $V_{p}^{\mathrm{w}}(f)$. Under the present definition of $V_{p}^{\mathrm{w}}(f)$, if $V_{p}^{\mathrm{w}}(f)=0$, we have such a sequence.

Let

$$
E_{f}(\delta, M)=\left\{x \in[0,1]: \limsup _{y \rightarrow x}|f(x)-f(y)||x-y|^{-\delta} \geqslant M\right\} .
$$

Lemma 3. If $E_{f}(\delta, M)$ has positive Lebesgue measure for some $M>0$, then $\operatorname{dim}_{\mathrm{s}}(f) \geqslant 1 / \delta$.

Proof. Suppose $\left|E_{f}(\delta, M)\right|>0$ for some $M>0$ and let $p<1 / \delta$. It is sufficient to show $V_{p}^{\mathrm{s}}(f)=\infty$. Let $\varepsilon>0$. For each $x \in E(\delta, M)$ there is an $h_{x}<\varepsilon$ such that

$$
\left|f(x)-f\left(x+h_{x}\right)\right| h_{x}^{-\delta} \geqslant M / 2 .
$$

The intervals $\left[x, x+h_{x}\right]$ cover $E_{f}(\delta, M)$ in the sense of Vitali, so there is a finite disjoint collection $\left\{\left[x_{1}, x_{1}+h_{1}\right], \ldots,\left[x_{N}, x_{N}+h_{N}\right]\right\}$ such that

$$
\left|E_{f}(\delta, M) \sim \bigcup_{i=1}^{N}\left[x_{i}, x_{i}+h_{i}\right]\right|<\frac{1}{2}\left|E_{f}(\delta, M)\right| .
$$

Now

$$
\begin{aligned}
V_{p}^{\mathrm{s}}(f) & \geqslant \sum_{i=1}^{N}\left|f\left(x_{i}\right)-f\left(x_{i}+h_{i}\right)\right|^{p}>\sum_{i=1}^{N} h_{i}^{\delta p}\left(\frac{M}{2}\right)^{p} \\
& >\left(\frac{M}{2}\right)^{p} \varepsilon^{\delta p-1} \sum_{i=1}^{N} h_{i}>\left(\frac{M}{2}\right)^{p} \varepsilon^{\delta p-1} \frac{1}{2}\left|E_{f}(\delta, M)\right|,
\end{aligned}
$$

which goes to $\infty$ as $\varepsilon \rightarrow 0$.

3. The functions of Besicovitch and Ursell. Besicovitch and Ursell [1] showed that if $f \in \operatorname{Lip} \delta$, then the Hausdorff dimension $d$ of the graph of $f$ satisfies $1 \leqslant d \leqslant 2-\delta$, and gave examples which showed that any $d$ in this interval is possible.

The function $f$ is defined as follows. Let $\varphi(x)=2 x$ for $0 \leqslant x \leqslant \frac{1}{2}$, and elsewhere define $\varphi$ by $\varphi(x)=\varphi(-x)=\varphi(x+1)$. Define $f$ by

$$
f(x)=\sum_{n=1}^{\infty} a_{n} \varphi\left(b_{n} x\right)
$$

where $a_{n}=b_{n}^{-\delta}$ and $b_{n+1}=b_{n}^{\mu_{n}}$.

For $\mu_{n}$, consider three cases.

(i) For $1<d<2-\delta, \mu_{n}=\mu=(1-\delta) \delta^{-1}(2-d)(d-1)^{-1}$. 
(ii) For $d=2-\delta, \mu_{n}$ is chosen so that $\mu_{n} \rightarrow 1$ and $b_{n+1} / b_{n} \rightarrow \infty$. (For example, $b_{1}>1$ and $\mu_{n}=1+n^{-1 / 2}$.)

(iii) For $d=1, \mu_{n}$ is chosen so that $b_{n} \rightarrow \infty$ and $\mu_{n} \rightarrow \infty$.

In (i) and (ii) we have

$$
\mu_{n} \geqslant \mu=(1-\delta) \delta^{-1}(2-d)(d-1)^{-1} .
$$

In (iii) we may assume

$$
\mu_{n} \geqslant(1-\delta) \delta^{-1}\left(2-d_{n}\right)\left(d_{n}-1\right)^{-1}, \quad \text { where } d_{n} \rightarrow 1 .
$$

In all three cases $b_{1}$ may be chosen so that

$$
b_{n+1} \geqslant B b_{n}, \quad \text { where } B>1 .
$$

LEMMA 4. For the function $f$ constructed above, there is an $M>0$ such that $E_{f}(\delta, M)=[0,1]$.

Proof. For any $x \in[0,1]$,

$$
\Delta f=f(x+h)-f(x)=\sum a_{n}\left[\varphi\left(b_{n} x+b_{n} h\right)-\varphi\left(b_{n} x\right)\right] .
$$

Let

$$
s_{\nu}=\sum_{n<\nu} a_{n}\left[\varphi\left(b_{n} x+b_{n} h\right)-\varphi\left(b_{n} x\right)\right]
$$

and

$$
r_{\nu}=\sum_{n>v} a_{n}\left[\varphi\left(b_{n} x+b_{n} h\right)-\varphi\left(b_{n} x\right)\right]
$$

Then

$$
\begin{aligned}
\left|r_{\nu}\right| & \leqslant \sum_{n>\nu} a_{n}\left|\varphi\left(b_{n} x+b_{n} h\right)-\varphi\left(b_{n} x\right)\right| \leqslant \sum_{n>\nu} a_{n}=\sum_{n>\nu} b_{n}^{-\delta} \\
& \leqslant b_{\nu+1}^{-\delta}\left[1+B^{-\delta}+B^{-2 \delta}+\cdots\right] .
\end{aligned}
$$

We have

$$
\left|r_{\nu}\right| \leqslant b_{\nu+1}^{-\delta} /\left(1-B^{-\delta}\right) \leqslant b_{\nu}^{-\delta} B^{-\delta} /\left(1-B^{-\delta}\right) .
$$

Since $\left|\varphi^{\prime}\right|=2$ we have

$$
\varphi\left(b_{n} x+b_{n} h\right)-\varphi\left(b_{n} x\right)= \pm 2 b_{n} h \quad \text { if } b_{n} h \leqslant \frac{1}{2} .
$$

So

$$
s_{\nu}=\sum_{n \leqslant \nu} \pm 2 b_{n}^{1-\delta} h
$$

Suppose the last term in this sum is positive. Then 


$$
\begin{aligned}
s_{\nu} & \geqslant 2 h\left[-b_{1}^{1-\delta}-\cdots-b_{\nu-1}^{1-\delta}+b_{\nu}^{1-\delta}\right] \\
& \geqslant 2 h b_{\nu}^{1-\delta}\left[-B^{(\nu-1)(\delta-1)}-B^{(\nu-2)(\delta-1)}-\cdots-B^{(\delta-1)}+1\right] \\
& >2 h b_{\nu}^{1-\delta}\left[1-\sum_{n=1}^{\infty} B^{(\delta-1) n}\right] \\
& =2 h b_{\nu}^{1-\delta}\left[2-\left(1-B^{\delta-1}\right)^{-1}\right] .
\end{aligned}
$$

If the last term in (8) is negative, we would have $s_{\nu}$ less than the negative of the last expression above. In any case $\left|s_{\nu}\right| \geqslant 2 h b_{\nu}^{1-\delta}\left[2-\left(1-B^{\delta-1}\right)^{-1}\right]$.

Combining this with (7) and letting $h_{\nu}=\left(2 b_{\nu}\right)^{-1}$, we have

$$
|\Delta f| \geqslant\left|s_{\nu}\right|-\left|r_{\nu}\right| \geqslant 2^{\delta}\left[2-\left(1-B^{\delta-1}\right)^{-1}-B^{-\delta}\left(1-B^{-\delta}\right)^{-1}\right] h_{\nu}^{\delta} .
$$

The expression in brackets is positive if $B$ is sufficiently large. This is accomplished by choosing $b_{1}$ large. Since $h_{v}$ is arbitrarily small, the lemma is proved.

Lemmas 3 and 4 and relation (2) yield the following.

THEOREM 1. For the function $f, \operatorname{dim}_{\mathbf{s}}(f)=1 / \delta$.

LeMma 5. For the function $f, V_{p}^{\mathrm{w}}(f)=0$ for every $p>1 /(2-d)$.

Proof. From (8),

$$
\begin{aligned}
\left|s_{\nu}\right| & \leqslant \sum_{n \leqslant \nu} 2 b_{n}^{1-\delta} h \\
& \leqslant 2 h b_{\nu}^{1-\delta}\left[1+B^{\delta-1}+B^{2(\delta-1)}+\cdots+B^{(\nu-1)(\delta-1)}\right] \\
& \leqslant K_{1} h b_{\nu}^{1-\delta} .
\end{aligned}
$$

Combining this with (7) yields

$$
|\Delta f| \leqslant\left|s_{\nu}\right|+\left|r_{\nu}\right| \leqslant K_{1} h b_{\nu}^{1-\delta}+K_{2} b_{\nu+1}^{-\delta} .
$$

Similarly we obtain

$$
\omega(f ; x, x+h) \leqslant K_{1} h b_{\nu}^{1-\delta}+K_{2} b_{\nu+1}^{-\delta}
$$

by applying (9) to the interval whose endpoints are where $f$ attains its maximum and minimum in $[x, x+h]$.

Now let $h_{\nu}=b_{\nu}^{\delta-1} b_{\nu+1}^{-\delta}$. Partitioning $[0,1]$ into intervals of length $h_{\nu}$, the estimate of $V_{1 /(2-d)}^{\mathrm{w}}(f)$ is (for cases (i) and (ii))

$$
\begin{aligned}
h_{\nu}^{-1}\left[\omega\left(f ; x, x+h_{\nu}\right)\right]^{1 /(2-d)} & \leqslant h_{\nu}^{-1}\left[K_{1} b_{\nu}^{1-\delta} h_{\nu}+K_{2} b_{\nu+1}^{-\delta}\right]^{1 /(2-d)} \\
& \leqslant K_{3} b_{\nu}^{1-\delta} b_{\nu+1}^{\delta(1-d) /(2-d)} \leqslant K_{3} .
\end{aligned}
$$

Now if $p>1 /(2-d)$,

$$
V_{p}^{\mathrm{w}}(f) \leqslant h_{\nu}^{-1}\left[\omega\left(f ; x, x+h_{\nu}\right)\right]^{p} \leqslant K_{3}\left[\omega\left(f ; x, x+h_{\nu}\right)\right]^{p-1 /(2-d)},
$$

which converges to zero as $h_{\nu}$ goes to zero. For case (iii) repeat the above 
argument replacing $d$ by $d_{n}$. Then if $p>1, p>1 /\left(2-d_{n}\right)$ for some $n$, and we again obtain $V_{p}^{\mathrm{w}}(f)=0$.

Lemma 5 and relation (3) yield the following.

TheOREM 2. For the function $f, \operatorname{dim}_{\mathrm{w}}(f)=1 /(2-d)$.

Note that as $d$ varies from 1 to $2-\delta, \operatorname{dim}_{\mathrm{w}}(f)$ varies from 1 to $1 / \delta$. In view of relations (2) and (3), Theorems 1 and 2 show that $f$ has the largest possible strong variation dimension and the smallest possible weak variation dimension.

\section{REFERENCES}

1. A. S. Besicovitch and H. D. Ursell, Sets of fractional dimension. V: On dimensional members of some continuous curves, J. London Math. Soc. 12 (1937), 18-25.

2. C. Goffman and J. Loughlin, Strong and weak $\Phi$-variation of Brownian motion, Indiana Univ. Math. J. 22 (1972/73), 135-138. MR 45 \#5288.

3. S. J. Taylor, Exact asymptotic estimates of Brownian path variation, Duke Math. J. 39 (1972), 219-241. MR 45 \#4500.

4. L. Yoder, The Hausdorff dimensions of the graph and image of $N$-parameter Brownian motion in d-space, Ann. Probability 3 (1975), 169-171.

5. — Variation of multiparameter Brownian motion, Proc. Amer. Math. Soc. 46 (1974), 302-309.

Department of Mathematics, University of Hawail, Honolulu, Hawail 96822 